Basic Health Sciences

Poster

Abstract ID: 135

\title{
Histological changes of uterus after comparative treatment of female rats by Trigonella foenum-graecum seeds and combined oral contraceptive pills
}

Ahmed Kaid Naji Allow | Ayah Rebhi Hilles | Zainab Yousef | Norbaiyah Mohamed Bakrim | Belqees Ahmed | Anil Kumar Saxena

Department of Basic Medical Sciences, Kulliyyah of Medicine, International Islamic University Malaysia

Introduction: This study evaluates the histological changes of uterine tissue in the female Sprague Dawley rats after administration of Trigonella foenum-graecum (fenugreek) seeds in comparison to combined oral contraceptive pills (COCPs). Methods: Twenty four female Sprague Dawley strain rats of 8 weeks old were divided into A, B, and C groups. Group A was the control and B was administrated with $0.05 \mathrm{mg} / \mathrm{kg}$ body weight of COCPs for 15 days. Group C was treated with $750 \mathrm{mg} / \mathrm{kg}$ body weight of fenugreek seed aqueous (FSA) extract for 15 days. The uterine tissues were isolated and studied histologically. Results: The uterine weight in the FSA extract treated group was normal, while in the COCPs treated group it was insignificantly increased. The endometrial thickness and endometrial gland density were normal in the FSA extract treated group, while in the COCPs treated group it was atrophied. Decidual-like cells were observed in the COCPs treated group only. Conclusions: Endometrial atrophy and decidual-like cells were noted after COCPs treatment while in the FSA extract and control groups they were absent. The findings suggest that fenugreek seeds could be used as an alternative natural and safe contraceptive product.

KEYWORDS: fenugreek seeds, combined oral contraceptive pills, contraception, endometrial atrophy 